\title{
Lavado de dinero y corrupción: necesidad de mayor investigación para un sustento empírico sólido*
}

\author{
[Artículos]
}

\author{
Martha Lucía Bahamón Jara** \\ Ximena Andrea Cujabante Villamil***
}

Alex Camilo Durán Montaño $o^{* * * *}$

Fecha de recepción: 4 de marzo de 2021

Fecha de aprobación: 18 de mayo de 2021

Citar como

Bahamón Jara, M. L., Cujabante Villamil, X. A. y Durán Montaño, A. C. (2021).

Lavado de dinero y corrupción: necesidad de mayor investigación para un sustento empírico sólido. Via Inveniendi Et Iudicandi, 16(2).

https://doi.org/10.15332/19090528.6781

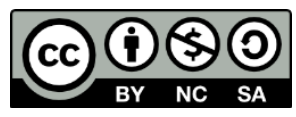

\footnotetext{
* Este artículo es producto de investigación del proyecto de investigación INV-EES 3174, titulado: "Crimen organizado y seguridad", financiado por la Universidad Militar Nueva Granada, Bogotá (Colombia)

** Magíster en Defensa de los Derechos Humanos y del Derecho Internacional Humanitario ante Organismos, Cortes y Tribunales Internacionales y especialista en Derecho Administrativo de la Universidad Santo Tomás. Abogada de la Universidad Libre de Colombia. Investigadora adscrita al grupo de investigación Sociedad, Estrategia y Seguridad. Docente tiempo completo de carrera de la Facultad de Relaciones Internacionales, Estrategia y Seguridad de la Universidad Militar Nueva Granada, Bogotá, Colombia. Correo electrónico: martha.bahamon@unimilitar.edu.co; ORCID: https://orcid.org/0000-0002-5877-6886

*** Doctora en Estudios Políticos de la Universidad Externado de Colombia y magíster en Asuntos Internacionales de la misma casa de estudios. Especialista en Negociación y Relaciones Internacionales de la Universidad de los Andes. Politóloga de la Pontificia Universidad Javeriana. Docente de tiempo completo de la Facultad de Relaciones Internacionales, Estrategia y Seguridad de la Universidad Militar Nueva Granada. Correo electrónico:

ximena.cujabante@unimilitar.edu.co; ORCID: https://orcid.org/0000-0002-5473-163X

**** Politólogo de la Universidad de los Andes, asistente de investigación en la Universidad Militar Nueva Granada, Bogotá, Colombia. Correo electrónico: ac.duran1035@uniandes.edu.co; ORCID: https://orcid.org/000-0001-9701-1520
}

Via Inveniendi Et Iudicandi

e-ISSN: 1909-0528 | DOI: https://doi.org/10.15332/19090528

Vol. 16 N.o 2 | julio-diciembre del 2021 


\section{Resumen}

El presente artículo analiza la literatura científica sobre lavado de dinero y corrupción producida durante los últimos quince años.

Específicamente, el artículo expone los principales hallazgos, consensos y vacíos de conocimiento de la literatura que versa sobre las definiciones y categorías de lavado de dinero y corrupción, y de las iniciativas legislativas, jurídicas y políticas orientadas a su combate, incluyendo iniciativas desde el sector privado o que comprendan modalidades de cooperación internacional. El artículo encuentra que si bien existen múltiples consensos respecto del impacto político, económico y social del lavado de dinero y la corrupción y la necesidad de emprender una lucha contra estos delitos de manera multidimensional e incluyendo al sector privado, existen dificultades sustanciales relacionadas a la comprensión real de los factores socioculturales e institucionales y los incentivos de economía política o los flujos de capitales transnacionales que ayudarían a entender la complejidad respecto de cómo ocurren estos delitos en la práctica. Lo anterior supone una preocupación apremiante, toda vez que se están implementado políticas sin un sustento empírico sólido.

Palabras clave: lavado de dinero, corrupción, políticas, sector privado, cooperación.

\section{Money laundering and corruption: need for further research for a solid empirical support}

\section{Abstract}

This article analyzes the scientific literature on money laundering and corruption produced during the last fifteen years. Specifically, the article presents the main findings, consensus and knowledge gaps in the literature on the definitions and categories of money laundering and corruption, and the legislative, legal and political initiatives aimed at fighting them, including initiatives from the private sector or involving international cooperation. The article finds that, although there are multiple consensus regarding the political, economic and social impact of

Via Inveniendi Et Iudicandi

e-ISSN: 1909-0528 | DOI: https://doi.org/10.15332/19090528

Vol. 16 N.० 2 | julio-diciembre del 2021 
money laundering and corruption and the need to undertake a multidimensional fight against these crimes, including the private sector, there are substantial difficulties related to real understanding of the sociocultural and institutional factors and the political economy incentives or transnational capital flows that would help to understand the complexity of how these crimes occur in practice. This is a pressing concern, since policies are being implemented without a solid empirical support.

Keywords: money laundering, corruption, policies, private sector, cooperation.

\section{Introducción}

Los vínculos entre el lavado de dinero y otros delitos o amenazas transnacionales han adquirido mayor atención en años recientes, tanto en la literatura académica como a nivel político. El lavado de dinero es un fenómeno que ha despertado interés en la literatura, la cultura popular y entre las autoridades desde la década de 1930 (Stessens, 2000) y, aunque a lo largo del tiempo se publicaron múltiples estudios que enfatizaron en el estatus del lavado de dinero como un asunto de importancia global y una amenaza seria (van Duyne, Harvey y Gelemorova, 2018), solo hasta 1989 se constituyó el Grupo de Acción Financiera Internacional (Financial Action Task Force), ampliamente reconocido como el primer esfuerzo por establecer estándares y promover la implementación efectiva de medidas operacionales, regulatorias y legales para combatir el lavado de dinero (Cox, 2014). Si bien la génesis del GAFI concebía el lavado de dinero como un crimen estrechamente ligado al tráfico de estupefacientes, durante las últimas décadas esta comprensión se ha ampliado para incluir otros delitos. En paralelo, el conocimiento académico sobre el lavado de dinero ha incrementado. 
De 2001 a 2004, nuevos estándares para el combate contra la financiación del terrorismo fueron incluidos en la misión del GAFI. De esta manera, se extendió la misión de este organismo intergubernamental y se formularon nueve recomendaciones especiales (anexadas a las cuarenta originalmente formuladas en su fundación en 1989) para abordar el delito de financiación del terrorismo. Posteriormente, estas recomendaciones han sido expandidas nuevamente para combatir nuevas amenazas asociadas con el lavado de dinero, entre las cuales se incluye la financiación de la proliferación de armas de destrucción masiva, y un impulso por fomentar mayor transparencia y una lucha más férrea contra la corrupción. De igual forma, a partir de los esfuerzos pioneros de John Walker, Brigitte Unger y Elena Madalina Busuioic para esclarecer la magnitud del lavado de dinero a nivel global (Walker, 1995; Unger y Busuioic, 2007; Walker y Unger, 2009), múltiples estudios han abordado fenómenos intrínsecos al lavado de dinero, principalmente, la acumulación de riqueza criminal (van Duyne, 2003; Savona y Riccardi, 2015) y el impacto negativo (potencial o real) sobre variables económicas (Quirk, 1996; Tanzi, 1996; Quirk, 1997; Unger et ál., 2006; Masciandaro et ál., 2007; Arnone y Borlini, 2010). Además, en el seno de la Organización de las Naciones Unidas (ONU), múltiples convenciones se han llevado cabo y varias resoluciones se han formulado, por ejemplo, la Convención de las Naciones Unidas contra el Tráfico Ilícito de Estupefacientes y Sustancias Psicotrópicas (Convención de Viena, 1998), la Convención de las Naciones Unidas contra la Delincuencia Organizada Transnacional (Convención de Palermo, 2000), o las resoluciones 1267 (1999) y 1373 (2001) del Consejo de Seguridad de dicha organización, para arraigar la lucha contra estos delitos percibidos como estrechamente relacionados (ONU, 1998; CSNU, 1999; UNODC, 2000; CSNU, 2001). 
Aunque la agenda global contra el lavado de dinero se ha, supuestamente, ampliado para incluir múltiples delitos y amenazas, los desarrollos a nivel político muestran lo contrario (Palomares y Calonje, 2015). Una revisión de los compromisos políticos a nivel global sobre el lavado de dinero evidencia que el pensamiento y la acción alrededor de este fenómeno siguen firmemente ancladas en verlo como un producto del tráfico de estupefacientes y el terrorismo, en lugar de materializar el supuesto compromiso frente a mayor transparencia y una lucha férrea contra la corrupción.

Por un lado, aunque existe una plétora de fuentes legislativas y regulatorias con prescripciones para combatir el lavado de dinero, el cuerpo de investigaciones empíricas que permitan la identificación de patrones generales sobre el tiempo es escaso (Levi, 2014). Asimismo, al analizar específicamente las cuarenta recomendaciones del GAFI en su presente forma, solo las recomendaciones que versan sobre los debidos procesos y mantenimiento de registros o las personas políticamente expuestas tienen algún contenido tangencialmente ligado a los vínculos entre el ejercicio protervo de las funciones públicas, malversación de fondos y la necesidad de ocultar el origen corrupto de dichos fondos (Cox, 2014, pp. 22-39) ${ }^{1}$. A pesar de la ratificación prolífica de la Convención de las Naciones Unidas contra la Corrupción ${ }^{2}$ y las provisiones en su contenido que precisan medidas preventivas contra la corrupción y su criminalización, al igual que asistencia técnica, cooperación internacional y mecanismos de recuperación de bienes para combatirla, hasta el

\footnotetext{
${ }^{1}$ Las 38 recomendaciones restantes del GAFI abordan asuntos como la cooperación y coordinación nacional; la ofensa de lavado de dinero; medidas de incautación; la financiación del terrorismo y la proliferación de armas; medidas de prevención; controles y grupos financieros; reportes de transacciones sospechosas; profesiones y negocios designados como no-financieros; propiedad de personas y arreglos legales; responsabilidades de autoridades competentes y medidas institucionales; asuntos operaciones y de fuerza pública; estadísticas; retroalimentación; sanciones, y cooperación internacional.

${ }^{2}$ A la fecha, solamente doce Estados miembro de la ONU aún no han ratificado esta convención.
} 
momento, los países que han ratificado la convención apenas están identificando sus necesidades de asistencia técnica, dados los obstáculos burocráticos y, en algunos contextos, por la ausencia de voluntad política (Lohaus, 2019).

Este compromiso político superficial por vincular el combate contra el lavado de dinero con aquel contra la corrupción parece fundamentarse en una tendencia aparentemente común en el campo del crimen transnacional: impulsar agendas políticas ambiciosas sin un arraigo en evidencia empírica sistemática. Pareciera que un interés súbito en casos notorios de corrupción en el Sur global (particularmente en países de África subsahariana) y la manera como múltiples formas de corrupción coadyuvan flujos voluminosos de bienes 3 produjo una visión en la cual los controles contra el lavado de dinero sirven como herramientas importantes para luchar contra la corrupción en estos países y, por extensión, la amenaza que pueden representar para los países de Occidente (Levi, 2014, p. 432). Varios autores han identificado esta tendencia de elucubrar objetivos políticos y movilizar recursos sin un trasfondo empírico y científico sólido en otros campos como el crimen organizado transnacional y el tráfico de drogas (Bewley-Taylor, 1999; van Duyne y Nelemans, 2011).

No obstante, la reticencia de actores políticos a fundamentar sus objetivos y compromisos en evidencia empírica y un sustento científico sólido no significa que este no exista. Como fue descrito anteriormente, los desarrollos políticos sobre el lavado de dinero se han realizado en tándem con desarrollos científicos en el estudio de dicho fenómeno. Cabe preguntarse cuáles son los vínculos entre el lavado de dinero y la corrupción en la literatura científica, y cómo podrían nutrir la agenda

\footnotetext{
${ }^{3}$ Ya sean ilegales (e.g., narcóticos), falsificados (e.g., software y propiedad intelectual, alcohol, medicinas, cigarrillos) o legales, pero evadiendo tributación.
}

Via Inveniendi Et Iudicandi

e-ISSN: 1909-0528 | DOI: https://doi.org/10.15332/19090528

Vol. 16 N. ${ }^{\circ} 2$ | julio-diciembre del 2021 
política ya existente. Por consiguiente, el presente artículo busca conectar la literatura que vincula explícitamente los fenómenos de lavado de dinero y corrupción, para mostrar cuáles son los factores, las condiciones, causas y los efectos que conectan estos dos delitos, y qué sugiere la evidencia científica respecto de posibles desarrollos políticos para combatir ambos fenómenos de manera efectiva.

El contenido restante de este artículo se estructura de la siguiente manera. En su marco teórico y conceptual, el artículo caracteriza la literatura producida sobre las categorías de lavado de dinero y corrupción, como aquella que aborda los impactos políticos y económicos de estos fenómenos. Posteriormente, la metodología del presente artículo describe brevemente la revisión sistemática de literatura desarrollada, incluyendo su lapso temporal, las bases de datos consultadas, los descriptores de búsqueda y los criterios de inclusión y exclusión del material bibliográfico revisado.

Más adelante, la sección de resultados del artículo reseña la producción académica que versa sobre los esfuerzos legislativos y la jurisprudencia para el combate del lavado de dinero y la corrupción; políticas y casos específicos de combate a estos delitos a nivel global; iniciativas en el sector privado que buscan combatir la corrupción y posterior esfuerzo por opacar la riqueza derivada de este y otros delitos; y los esfuerzos de cooperación internacional que propenden por combatir la corrupción y el lavado de dinero. A manera de discusión, el artículo plantea una serie de aseveraciones relacionadas al estatus actual de este cuerpo de conocimiento y sus implicaciones frente a desarrollos de política pública y posibles investigaciones a futuro. Por último, el artículo cierra con una serie de conclusiones que sintetizan su contenido principal. 


\section{Marco teórico y conceptual}

Llama la atención que, en medio del acervo de literatura académica que se ha producido sobre corrupción y lavado de dinero, pocos autores osan definir de manera precisa ambos fenómenos. De las fuentes revisadas para el presente artículo, las definiciones más específicas de "lavado de dinero" y “corrupción” son proferidas por John S. Zdanowicz (2009) y Roger Bate (2007), respectivamente. Por un lado, Zdanowicz define el "lavado de dinero", como el proceso de ocultar la existencia o aplicación de ingresos derivados de una actividad criminal, y el posterior encubrimiento de la fuente de dicho ingreso para hacerlo parecer legítimo (Zdanowicz, 2009, p. 855). Por otro lado, Bate define la "corrupción" como el uso de un cargo público para avanzar intereses o usufructos privados (Bate, 2007, p. 89). Si bien el cuerpo de literatura que conecta estos delitos es voluminoso, los textos de Zdanowicz y Bate son los que, de manera sistemática, establecen su significado antes de hablar sobre sus diferentes tipologías, la aparente conexión entre ellos o sus impactos a nivel político, económico y social (Mosquera y González, 2020).

Existe un esfuerzo desde la academia por caracterizar con la mayor fidelidad posible la manera en que ambos delitos se manifiestan. En su estudio circunscrito a Alemania, Austria y Suiza, Ronald Füss y Achim Hecker (2008) diferencian entre cinco tipos de crímenes de "cuello blanco", incluyendo la corrupción, el fraude, robo, la competencia desleal y el lavado de dinero. Con los criterios de frecuencia de ocurrencia y magnitud de daño, estos autores sitúan la corrupción y el lavado de dinero en una categoría de crímenes de cuello blanco denominada "tipo minoría", ya que señalan que son poco frecuentes en relación con otros crímenes de cuello blanco (e.g., fraude y robo), pero que su daño social es más grande (Fuss y Hecker, 2008, p. 155). Sin embargo, este estudio muestra una debilidad estructural de la producción académica sobre el lavado de dinero

Via Inveniendi Et Iudicandi

e-ISSN: 1909-0528 | DOI: https://doi.org/10.15332/19090528

Vol. 16 N.० 2 | julio-diciembre del 2021 
y la corrupción, en la que, si bien se enuncia que usualmente son delitos conectados y que ambos representan un impacto negativo de grandes dimensiones, no se ahonda sobre los factores causales o mecanismos que explican por qué son delitos casi inextricables.

Aun con esta debilidad, la cantidad de estudios que indagan sobre los efectos del lavado de dinero y la corrupción a nivel político, económico y social son sustanciales. Jan Van Dijk (2007), Luminita Ionescu (2010), Pramila Choudhary (2016), Stefan Bojnec e Imre Fertö (2018) y Ana Julia Akaishi Padula y Pedro Henrique Melo Albuquerque (2018) utilizan modelos econométricos, índices compuestos y casos de estudio para abordar los impactos predominantemente negativos de la corrupción $-\mathrm{y}$ el uso del lavado de dinero para ocultar las fuentes de ingresos derivados de la corrupción- sobre riqueza nacional, las conexiones políticascorporativas, el crecimiento económico, la inversión extranjera directa y los mercados de capitales (Ionescu, 2012a, p. 159). Estos autores indagan a fondo sobre los impactos de estos crímenes, pero sus estudios no profundizan en los factores específicos o los mecanismos causales que explican por qué la corrupción y el lavado de dinero ocurren simultáneamente, más allá de indicar que el lavado de dinero facilita la corrupción.

\section{Metodología}

Para la elaboración de este artículo, en materia metodológica, se realizó una revisión de literatura sistemática del material científico de acceso abierto sobre el lavado de dinero y la corrupción, producido desde el 2005 en adelante. Este año fue escogido como el inicio de la búsqueda de literatura dado que marca la aprobación de la Convención de las Naciones Unidas contra la Corrupción, entendido como el punto de convergencia entre la lucha global contra el lavado de dinero y el combate global contra 
la corrupción. Por medio de una búsqueda en tres de las principales bases de datos para la recolección de material bibliográfico (JSTOR®, EBSCOHost $\AA$, y Scopus $($ ), utilizando los términos de búsqueda money laundering y corruption (unidos a través del operador booleano AND). Se excluyó material aparte de artículos científicos revisados por pares (debido a los estándares de revisión y publicación en comparación con otro tipo de material bibliográfico) y se consideraron relevantes los artículos con referencias directas al lavado de dinero y a la corrupción en su título, resumen o lista de palabras clave.

A partir de dichos criterios empleados, de inclusión y exclusión, se configuró un conjunto de 41 artículos, conferencias y entrevistas revisados. Como observación metodológica, es importante resaltar que la revisión desarrollada en el marco de esta investigación es exploratoria. Por consiguiente, en lugar de aspirar a ser una revisión exhaustiva de la totalidad del material bibliográfico producido sobre el tema, la metodología busca proporcionar unos hallazgos iniciales y alimentar una discusión que proporcione unos elementos de análisis iniciales e interrogantes que conduzcan a la producción de mayor investigación y un diálogo académico y político dinámico sobre el lavado de dinero y corrupción.

\section{Resultados}

Existe un núcleo de estudios que ha abordado el lavado de dinero y la corrupción desde una perspectiva jurídica. Mientras que algunos de estos trabajos abordan posibles investigaciones y teorías generales sobre elementos cruciales en el campo legal, otros utilizan casos específicos a nivel global para ilustrar posibles mejoras en las prácticas, en lo referente al combate de estos dos delitos. 
Por un lado, Luminita Ionescu (2012a) plantea que existe una necesidad creciente de investigar los niveles percibidos de corrupción judicial en la Unión Europea, así como la complejidad detrás de la corrupción en diversas jurisdicciones, las causas de la corrupción en los sectores públicos y privados, y el impacto del internet sobre la corrupción, teniendo en cuenta la responsabilidad de personas jurídicas en el lavado de ingresos derivados de la corrupción (Ionescu, 2012a, p. 159). En paralelo, Sergio Salgado, Luis Oceja, Maité Beramendi y Carolina González (2018) profieren un nuevo modelo para entender la adherencia a las normas, informados por el estudio de los impactos directos de la violación regulatoria (por vía de corrupción y lavado de dinero) a nivel social y las dificultades de la administración normativa a nivel social y organizacional (Salgado et ál., 2018, p. 485).

Por otro lado, los estudios de Carl Pacini (2012) y Radha Dawn Ivory (2013) abordan prácticas jurídicas para combatir el lavado de dinero y la corrupción en Estados Unidos y la Unión Europea. Pacini sostiene que si bien administradores corporativos, oficiales financieros, miembros de juntas directivas, auditores y contadores forenses tienen claridad sobre los objetivos y mandatos de la Ley de Prácticas Corruptas en el Extranjero de Estados Unidos, un número significativo no protege adecuadamente sus firmas o clientes de los costos monetarios y no-monetarios de violar esta Ley, ya sea por vía de acción legal, investigaciones tributarias o indagaciones oficiales por lavado de dinero (Pacini, 2012, p. 545). En su análisis de la jurisprudencia de la Corte Europea de Derechos Humanos, en materia de órdenes de expropiación de bienes, relacionada con asuntos de lavado de dinero, Dawn Ivory (2013, p. 148) sostiene que la figura de la "negación flagrante de la justicia" ha exacerbado la cooperación en materia de recuperación de bienes malversados por corrupción. 
Otros estudios han intentado identificar mejores prácticas en otras latitudes, particularmente en Asia y América Latina. Noel G. Villaromán (2012), por un lado, explica la manera en la cual la Ley de Riqueza no Explicada en Filipinas previene conflictos jurisdiccionales entre la Oficina de la Personería y el Concejo Antilavado de Dinero de la Procuraduría en ese país (Villaromán, 2012, p. 749). Por su parte, Luiz Regis Prado (2013) ilustra cómo la decisión de castigar penalmente el blanqueo de capitales en Brasil se queda corto frente a la realidad del delito, puesto que trata la corrupción y el lavado de dinero como delitos independientes (Regis Prado, 2013, p. 398), dificultando su procesamiento judicial efectivo y las capacidades de investigación.

Por su parte, la literatura sobre medidas políticas para combatir el lavado de dinero y corrupción se puede organizar en dos niveles de análisis, de manera similar al núcleo de estudios abordado en la sección anterior. En primer lugar, existen varios estudios que profieren planteamientos teóricos sobre cómo movilizar recursos y formular políticas públicas para reducir la corrupción, el lavado de dinero y sus impactos. Además, diversos estudios exploran desafíos, prácticas, impactos y lecciones de esfuerzos en América del Norte, Europa Occidental, Europa del Este y Asia Central, África y Asia del Sur.

A nivel teórico, la producción académica sobre políticas para combatir el lavado de dinero y la corrupción se centran sobre los impactos del crimen organizado sobre la seguridad nacional, el sustento desde la econometría para el diseño de medidas que reduzcan la corrupción, y el concepto de disuasión. Mientras que Alin Pahontu (2010) sostiene que el lavado de dinero y la corrupción debilitan las instituciones estatales y generan una pérdida de la confianza ciudadana en el imperio de la ley, perjudicando fundamentalmente los cimientos económicos y democráticos de la sociedad (Pahontu, 2010), Armey y Melese (2018) explican -al estudiar 
qué iniciativas se pueden tomar tanto en el sector público como en el privado- que un marco probabilístico de optimización señala que hay tres tipos de medidas que son efectivas para reducir la corrupción y el lavado de dinero, a saber: (i) "construcción de integridad" por medio de códigos de conducta y capacitaciones al interior de las organizaciones para reducir la utilidad individual que pueda vislumbrarse en actividades delictivas; (ii) "incremento de transparencia" invirtiendo en sistemas contables, control de inventarios y auditorías independientes que aumenten la probabilidad de detección y castigo de actividades delictivas; y, (iii) "mejoramiento de la rendición de cuentas" a través de mejoras al sistema judicial y legal que incremente la posibilidad de condena y consecuencias para los condenados de estos delitos (Armey y Melese, 2018, pp. 847-848). Posteriormente, Moh Zali y Ach Maulidi (2018), centrándose sobre el caso de los países BRIC (Brasil, Rusia, India y China), sostienen que para contrarrestar la complejidad y opacidad en casos de lavado de dinero para blanquear ingresos de la corrupción es necesario potenciar la administración pública y veeduría ciudadana sobre sistemas transfronterizos de transacción, para aumentar las sanciones informales o sociales que puedan servir para disuadir el delito en conjunto con programas públicos (Zali y Maulidi, 2018, pp. 60-61).

Para resaltar las medidas implementadas en Estados Unidos y Europa Occidental, Josh O. Ungerman (2013) y Luminita Ionescu (2012b) hacen hincapié en las ventajas de la expansión del mandato del Servicio de Impuestos Internos (IRS, por sus siglas en inglés) de Estados Unidos y las Directivas contra el Lavado de Dinero de la Unión Europea, respectivamente. Ungerman (2013) señala como una ventaja el hecho de que el IRS se involucre de manera activa en investigar casos de corrupción pública y el empleo de técnicas de cálculo de escenarios para identificar estructuras de recuperación de bienes (Ungerman, 2013, p. 28). Por su

Via Inveniendi Et Iudicandi

e-ISSN: 1909-0528 | DOI: https://doi.org/10.15332/19090528

Vol. 16 N.० 2 | julio-diciembre del 2021 
parte, Ionescu (2012b, p. 562) ha resaltado la manera en la que la Unión Europea ha adoptado tres directivas sucesivas - abordando asuntos diversos como el combate de ganancias del tráfico de drogas, el reporte de transacciones sospechosas en las que se estime la ocurrencia de cualquier delito y la responsabilidad de todo profesional y actividades no-financieras como abogados, notarios, contadores y agentes hipotecarios- para facilitar la implementación de las recomendaciones del GAFI.

Además, otra parte de la literatura sobre políticas orientadas a combatir el lavado de dinero y la corrupción se circunscribe a casos de Europa del Este y Asia Central, donde se resaltan primordialmente las dificultades políticas de combatir estos delitos. En primer lugar, Burger (2009) enumera los desafíos para el establecimiento de un sistema efectivo contra el lavado de dinero en Rusia, a saber: la falta de desarrollo en el sector bancario, dado que las instituciones financieras existen principalmente para proveer servicios a las corporaciones enormes de las que son propiedad; la falta de personal, bienes materiales y apoyo político a las entidades burocráticas encargadas del combate contra el lavado de dinero; la complejidad de transacciones en las que corporaciones rusas están involucradas; la corrupción integral a los diferentes niveles de gobierno; la sofisticación de los grupos de crimen organizado; y la inestabilidad económica (Burger, 2009, p. 41). Por otro lado, Erica Marat (2015) resalta que la malversación sistemática de fondos durante el régimen Bakiyev en Kirguistán fue facilitada por la falta de regulación en el sector financiero para prevenir la deslocalización de fondos, aunando el apoyo político informal de algunos poderes regionales y globales (Marat, 2015, pp. 54-55). De igual manera, Kostyuchenko et ál. reconocen la corrupción dominante en Ucrania y sugieren acompañar políticas estatales contra la corrupción con evaluación de impacto efectiva, particularmente para minimizar los flujos opacos de fondos en las economías; las autoras recomiendan implementar esta

Via Inveniendi Et Iudicandi

e-ISSN: 1909-0528 | DOI: https://doi.org/10.15332/19090528

Vol. 16 N.० 2 | julio-diciembre del 2021 
evaluación con diversos grados de intensidad según los diferentes sectores de la economía, dependiendo del nivel de riesgo determinado por el tipo de actividad económica, las autoridades involucradas, y los países de origen y destino (Kostyuchencko et ál., 2018, p. 41).

En el caso de África, la literatura que estudia el lavado de dinero y la corrupción se centra notoriamente en el caso de Nigeria. Primero, Emmanuel Obuah (2010) sostiene que la Comisión de Crímenes Financieros y Económicos, el cuerpo ejecutivo designado para combatir el lavado de dinero y la corrupción en ese país, se enfrenta a múltiples desafíos en su gestión por la naturaleza incierta del panorama político nigeriano y la manera en que dicho panorama suscita la extracción de rentas por parte de partidos e instituciones políticas (Obuah, 2010, p. 17). Segundo, David U. Enweremadu (2013) explica que los intentos por recuperar bienes malversados, como aquellos lanzados en Nigeria luego de la transición a la democracia en 1999, con el propósito de recuperar los fondos públicos en cuentas extranjeras, malversados por el régimen Abacha, son obstaculizados por la falta de voluntad política a nivel internacional y por una falta de capacidades legales y contables, así como también por la falta de voluntad política a nivel doméstico (Enweremadu, 2013, p. 51). Por último, años después, se advirtió sobre el riesgo presentado por el hecho de que varios parlamentarios investigados por la Comisión de Crímenes Financieros y Económicos, que lograron presentar estratagemas y dilaciones en sus procesos, pudieran participar activamente del proceso electoral y la elección presidencial programada para 2015, ya sea directamente o a través de terceros (Oji et ál., 2014, p. 207).

Para concluir, en la literatura dedicada al estudio de los dos delitos en Asia predomina el análisis sobre la reciente medida de desmonetización en India, donde el gobierno del primer ministro, Narendra Modi, decidió

Via Inveniendi Et Iudicandi

e-ISSN: 1909-0528 | DOI: https://doi.org/10.15332/19090528

Vol. 16 N.o 2 | julio-diciembre del 2021 
desmonetizar los billetes de grandes denominaciones ( $₹ 500$ y ₹ 1000) y reemplazarlos en un intento por reducir el uso de dinero falso o de origen dudoso para financiar actividades ilegales. Por un lado, Jiten Kumar Mishtra (2017) sostiene que el impacto de esta política fue devastador por la reducción de la liquidez en la economía, paralizando el sector informal, del cual una gran cantidad de indios dependían para su bienestar (Kumar Mishtra, 2017, p. 53), y otros autores han manifestado críticas a la política, ya sea por la facilidad de injerencia de grupos de interés domésticos e internacionales en su formulación o por el desempleo, los suicidios y la reducción de salarios diarios como resultado de su implementación, interpretada como intempestiva y con falta de un proceso cuidadoso de planeación (Goyal y Singh, 2019; Bose, 2019). En contraste, autores como Fathima Sumaiya (2018) han apoyado la política, porque se espera un mejoramiento en el déficit nacional y menos corrupción y lavado de dinero en el ámbito empresarial a raíz de su implementación (Sumaiya, 2018, p. 20).

A pesar de la importancia de la adherencia del sector privado a esfuerzos para combatir la corrupción y el lavado de dinero, la literatura sobre la participación de organizaciones privadas o las iniciativas que han emprendido en esta materia es escasa. No obstante, los trabajos existentes en este campo enfatizan la existencia simbiótica de corrupción a nivel público y privado, y detallan algunos mecanismos de defensa que las empresas privadas han configurado para combatir contra estos delitos. Ed Brown y Jon Cloke (2007) refutan la visión de la corrupción como un caso de aberraciones individuales en una economía global funcional, al introducir el concepto de Europa ensombrecida. Bajo este concepto, los autores explican la manera en que la noción de corrupción suele limitarse, erróneamente, a países del Sur global donde un Estado gigantesco engendra fallas de mercado, y que, en realidad, en Europa Occidental

Via Inveniendi Et Iudicandi

e-ISSN: 1909-0528 | DOI: https://doi.org/10.15332/19090528

Vol. 16 N.o 2 | julio-diciembre del 2021 
misma han existido toda una serie de conflictos de interés, lavado de dinero y casos de corrupción política por parte de gobiernos y corporaciones privadas que han "normalizado" la coexistencia entre la legalidad e ilegalidad en la economía de esa región. Por consiguiente, los autores plantean que la corrupción debe ser conceptualizada como un instrumento esencial de acumulación de capital y política con igual prevalencia en los sectores públicos y privados, para permitir una evaluación honesta del funcionamiento y magnitud de la corrupción que, a su vez, facilite su combate (Brown y Cloke, 2007, p. 305).

Otros autores explican los mecanismos de defensa que las empresas privadas han desarrollado para cumplir con mandatos internacionales y locales para combatir el lavado de dinero y corrupción. Por un lado, las empresas privadas han desarrollado funciones de tesorería para prevenir y detectar riesgos de lavado de dinero y corrupción, alinear sus procesos operativos, contables y ejecutivos con los mandatos de la Ley de Prácticas Corruptas en el Extranjero, y optimizar la integridad, supervisión y diligencia debida de socios y prestadores de servicios, además de arraigar procesos permanentes de evaluación de resultados y efectividad de estas medidas (Jourdan et ál., 2008, p. 247). A su vez, estas medidas están arraigadas en el concepto de conformidad, que buscan brindar una respuesta institucional a la inseguridad de un ambiente corporativo marcado por riesgos de no cumplimiento ante un andamiaje normativo cada vez más complejo (de Courcelles, 2009, p. 30). En uno de los pocos casos empíricos pertenecientes a este núcleo de literatura, Diana Ester Albanese (2012) analiza la manera en la cual, tras deliberaciones a nivel administrativo, la implementación de una matriz de riesgo en un banco de Bahía Blanca (provincia de Buenos Aires, Argentina) catalizó la instauración de una cultura de control y alerta de operaciones inusuales y 
sospechosas, y los rudimentos de una política de "conocer a tu cliente" (Albanese, 2012, p. 206).

Adicionalmente, existe un núcleo de literatura que ha abordado la necesidad de aumentar y refinar la cooperación internacional para combatir el lavado de dinero y la corrupción. Aunque inicialmente la literatura se concentró sobre la necesidad que dicha cooperación se consolidara entre países de África y de otros continentes (particularmente Europa), se ha ampliado el foco de análisis progresivamente para abordar otros tipos de cooperación.

Los trabajos de Penny Jackson (2005) y Sandy Robertson (2006), por un lado, hicieron hincapié sobre la necesidad de escudriñar el rol de países de Occidente en el pago de sobornos y lavado de dinero (particularmente a través de agencias de exportación), y de aumentar el procesamiento judicial y la condena de casos de corrupción y malversación de fondos en países donantes de ayuda internacional a África, respectivamente (Jackson, 2005; Robertson, 2006). Otros trabajos han ampliado este foco de análisis para argumentar que se necesita más cooperación Norte-Sur para combatir efectivamente el lavado de dinero y la corrupción.

Uno de estos trabajos sostiene que, si bien se han desarrollado y difundido innumerables sistemas de antilavado de dinero alrededor del mundo - específicamente a países en vías de desarrollo-, hay un déficit de apropiación local de estos sistemas, lo cual genera que de por sí la simple exportación de sistemas eficientes no es suficiente para combatir estos delitos (Sharman y Chaikin, 2009, p. 27). Igualmente, otros autores han encontrado que en algunos casos los usufructos "lavados" de la corrupción son usados para adquirir bienes ilegales, y que debe existir mayor coordinación entre los mercados exportadores e importadores de estos bienes para diseñar estrategias de control, como es el caso en el comercio de árboles talados ilegalmente (Kishor y Lescuyer, 2012). Por último, 
Sungyong Kang (2018) resalta los avances recientes en materia de coordinación entre países del Norte global con aquellos en vías de desarrollo (como también entre organizaciones públicas y privadas) para homogeneizar sus bases de datos de personas políticamente expuestas, aunque advierte que esa cooperación debe dar lugar a que se pueda conminar a las instituciones financieras a compartir su información con autoridades públicas para mayor control e identificación quirúrgica de los casos en los que se presentan los delitos (Kang, 2018, pp. 719-720).

Otros autores han indagado sobre la relevancia de cooperación bilateral e intrarregional en este aspecto. En primer lugar, Gong Xiangqian (2013) aboga por investigaciones conjuntas de malversación de fondos, sobornos y lavado de dinero entre el gobierno chino y las autoridades canadienses para, eventualmente, recuperar y distribuir conjuntamente bienes recuperados (Gong, 2013, p. 594). Por otro lado, mientras que Cyrille Fijnaut (2013) aduce las fallas de la Comisión Especial contra el Crimen Organizado del Parlamento Europeo (la Comisión Alfano) a la desconexión entre las interpretaciones de países miembro de la Unión Europea sobre el crimen organizado, la corrupción y el lavado de dinero (Fijnaut, 2013, p. 4), Francesco Calderoni (2015) señala asimetrías regulatorias entre países de la Unión Europea (particularmente entre países continentales y aquellos offshore) como la fuente de mayor opacidad en las transacciones financieras en el continente (Calderoni, 2015, p. 135).

\section{Discusión}

En primer lugar, los estudios relacionados con esfuerzos legislativos y la jurisprudencia para el combate del lavado de dinero y la corrupción profieren toda una serie de lecciones y sugerencias para iniciativas legislativas y judiciales que propendan por el combate de la corrupción y el lavado de dinero. Sin embargo, si bien recalcan las fortalezas o debilidades 
de algunos de los instrumentos jurídicos existentes, estos estudios no parecieran ofrecer un panorama detallado de los mecanismos o factores causales detrás de la ocurrencia de la corrupción y el lavado de dinero de manera inextricable, ni de las dimensiones que sugieren aquellos estudios seminales que plantean la urgencia de combatir la ocurrencia conjunta de estos delitos por sus impactos multidimensionales y su magnitud gigantesca a nivel global.

El siguiente compendio de literatura revisado arroja varias lecciones sobre los esfuerzos políticos a nivel global por combatir el lavado de dinero y la corrupción. Primero, los casos de Estados Unidos y la Unión Europea recalcan la importancia de ampliar los mandatos de las unidades de inteligencia financiera y servicios de aduanas para fortalecer las capacidades investigativas en casos de corrupción política, como también de crear directivas que faciliten la apropiación local y operacionalización de mandatos internacionales como las recomendaciones del GAFI. Segundo, los casos de Europa del Este, Asia Central y África ilustran la necesidad de implementar esquemas de capacitación contable y financiera para órganos estatales dedicados al combate del lavado de dinero y la corrupción, y de acompañar lo anterior con una movilización sustancial de recursos, apoyo político, y un aumento tanto de la capacidad legal de los cuerpos de investigación como de evaluaciones de impacto para determinar la viabilidad y los resultados de estas políticas, y de ser necesario, reorientar sus enfoques, operatividad o ejecución presupuestal. Para terminar, el caso de la desmonetización en India muestra la necesidad de acompañar políticas que propenden por luchar contra la corrupción y el lavado de dinero con una planeación a mediano y largo plazo, y una evaluación de riesgo frente a la economía nacional. Sin embargo, a pesar de todas estas lecciones, la literatura no contiene detalles sobre cómo operan en conjunto estos delitos en los casos abordados, y los 
autores no desglosan la complejidad de la corrupción y el lavado de dinero en los países estudiados.

Por su parte, el núcleo de la literatura sobre el lavado de dinero y corrupción en el sector privado muestra que estos delitos no se circunscriben necesariamente al sector público, ni a países en vías de desarrollo. De igual forma, se ilustran los esfuerzos desde la empresa privada por incrementar la capacidad de evaluar, monitorear y reportar riesgos. Sin embargo, se necesita un mayor número de estudios empíricos para identificar las percepciones y los discursos que se forman en el personal de las empresas frente a los mecanismos detrás de estos delitos y cómo combatirlos mejor, que se pueda utilizar para informar mejor sobre procesos de formulación, implementación y seguimiento a políticas públicas que busquen luchar de manera integral contra estos delitos. A pesar de abordar distintos niveles de cooperación, las fuentes relacionadas a ese tema en particular abogan en unísono por mayor concertación, cooperación y diálogo entre los países para combatir el lavado de dinero y corrupción. Sin embargo, existe una ausencia de estudios empíricos que analicen la manera como estos delitos transcurren a nivel transfronterizo y cómo se adaptan a marcos regulatorios disímiles para suministrar un sustento científico empírico para informar cualquier intento por aumentar la cooperación y efectividad de la lucha conjunta contra estos delitos.

Si bien la discusión sobre las fuentes revisadas arroja algunos consensos y evidencia una década y media de producción académica constante, existe un déficit de evidencia empírica que discierna los diversos factores causales, riesgos y mecanismos mediante los cuales el lavado de dinero y la corrupción operan en conjunto y manifiestan sus impactos. De los estudios analizados, como aquellos trabajos seminales antes revisados, se desprende un sentido de urgencia y un entendimiento superficial sobre la

Via Inveniendi Et Iudicandi

e-ISSN: 1909-0528 | DOI: https://doi.org/10.15332/19090528

Vol. 16 N.० 2 | julio-diciembre del 2021 
ocurrencia simultánea de la corrupción y el lavado de dinero, pero no se ha constituido aún un análisis integral sobre los factores socioculturales, institucionales, los incentivos de economía política o los flujos de capitales transnacionales que coadyuvan la corrupción y el uso del lavado de dinero para blanquear el origen de estos fondos malversados para su usufructo por parte de individuos y grupos. De esta manera, resulta crítico generar evidencia científica para contribuir a la comprensión de los elementos anteriormente listados, puesto que solo entendiendo cabalmente la economía política del lavado de dinero y la corrupción es posible informar con una base empírica sólida esfuerzos de formulación de política en los países donde estos delitos son desafíos más apremiantes, o iniciativas de diseño institucional que generen recomendaciones y esquemas para asistir a los esfuerzos de lucha conjunta contra los delitos a nivel global.

\section{Conclusión}

En el presente artículo se ha realizado una revisión de una porción significativa de la literatura académica sobre el lavado de dinero y corrupción. Esta aproximación ayuda a comprender mejor, de manera integral, los temas, consensos y vacíos que presenta la literatura en cuanto al entendimiento de estos dos delitos, la manera como se manifiestan en la práctica, y qué se puede hacer para combatirlos. Particularmente, se ha puesto énfasis sobre las categorías que emergen desde la literatura para definir y categorizar las maneras como el lavado de dinero y la corrupción se materializan en la práctica, las diferentes medidas que se pueden adoptar en diferentes niveles de poder (legislativo, judicial, ejecutivo) para combatir estos delitos y los impactos de dichas medidas, y cómo se vislumbra el rol del sector privado y el potencial de cooperación en múltiples niveles, como parte de un esfuerzo multisectorial y 
multidimensional por mitigar la dimensión y los efectos globales de ambos delitos.

Analizando la literatura que define y categoriza el lavado de dinero y la corrupción, se encontró que existe un consenso científico en relación con los impactos negativos a nivel político, económico y social de ambos delitos, pero que no existe claridad sobre cómo definir sucintamente ambos delitos o la magnitud de sus impactos a nivel global.

Posteriormente, la literatura sobre medidas legislativas, normativas y políticas para combatir el lavado de dinero y la corrupción muestra que si bien existen leyes alrededor del mundo que enfatizan la ocurrencia en conjunto de estos delitos y que potencian las capacidades tecnológicas, investigativas, judiciales y administrativas para su combate, aún existe una necesidad apremiante de mayor capacitación técnica y tecnológica, mayor apropiación y ejecución de recursos monetarios y no-monetarios, mayor voluntad política, evaluación rigurosa de impacto y riesgos de las medidas y planeación a mediano y largo plazo para contar con leyes, órganos y políticas que combatan el lavado de dinero y la corrupción de manera eficaz y eficiente. Por último, la literatura sobre iniciativas del sector privado y cooperación demuestra que, a pesar de los desafíos enumerados, existe un consenso naciente sobre la prevalencia de estos delitos, tanto en el sector privado como el público, y la necesidad de diseñar esquemas efectivos de cooperación a nivel bilateral y multilateral -incluyendo esfuerzos intrarregionales- para luchar de manera integral contra estos dos delitos y lograr los resultados esperados y necesarios para evitar un mayor detrimento político, económico y social a nivel global.

En resumen, lo que parece claro de este análisis es que los efectos del lavado de dinero y la corrupción parecen de importancia central para tomadores de decisión y analistas alrededor del mundo, particularmente en países donde ambos delitos representan amenazas mayúsculas a la vida 
política, económica y el bienestar social. Pero nuestra comprensión de estos delitos sigue siendo limitada, por lo cual el tema amerita mayor investigación.

\section{Referencias}

Albanese, D. E. (2012). Análisis y evaluación de riesgos: aplicación de una matriz de riesgo en el marco de un plan de prevención contra el lavado de activos. BASE, 9(3), 206-215. https://doi.org/10.4013/base.2012.93.01

Armey, L. y Melese, F. (2018). Minimizing Public Corruption: The Economics of Crime, Identity Economics, and Money Laundering. Defence and Peace Economics, 29(7), 840-852. https://doi.org/10.1080/10242694.2017.1318013

Akaishi Padula, A. J. y Melo Albuquerque, P. H. (2018). Government Corruption on Brazilian Capital Markets: A Study on Lava Jato (Car Wash) Investigation. Revista de Administracao de Empresas (Journal of Business Management), 58(4), 405417. https://doi.org/10.1590/So034-759020180406

Arnone, M. y Borlini, L. (2010). International anti-money laundering programs. Empirical assessment and issues in criminal regulation. Journal of Money Laundering Control, 13(3), 226-271. https://doi.org/10.1108/13685201011057136

Bate, R. (2007). Unhealthy Corruption. Economic Affairs, 27(1), 89-89. https://doi.org/10.1111/j.1468-0270.2007.00720.x

Bewley-Taylor, D. R. (1999). The United States and international drug control, 19091997. Pinter.

Bojnec, S. y Fertö, I. (2018). Globalization and Outward Foreign Direct Investment. Emerging Markets, Finance \& Trade, 54(1), 88-99. https://doi.org/10.1080/1540496X.2016.1234372

Bose, F. (2019). An Economic and Public Policy View of Demonetization in India. Society, 56(1), 38-46. https://doi.org/10.1007/s12115-018-00322-9

Burger, E. S. (2009). Following Only Some of the Money in Russia. Demokratizatsiya, 4170, Invierno 2009, Georgetown Law and Economics Research Paper n. ${ }^{\circ}$ 10-01. Disponible en: https://ssrn.com/abstract $=1543240$.

Via Inveniendi Et Iudicandi e-ISSN: 1909-0528 | DOI: https://doi.org/10.15332/19090528 Vol. 16 N.० 2 | julio-diciembre del 2021 
Brown, E. y Cloke, J. (2007). Shadow Europe: Alternative European Financial

Geographies. Growth and Change, 38(2), 304-327. https://doi.org/10.1111/j.14682257.2007.00368.x

Calderoni, F. (2015). The analysis and containment of organized crime and transnational organized crime: an interview with Ernest U. Savona. Trends in Organized Crime, 18(1), 128-142. https://doi.org/10.1007/s12117-014-9232-x

Choudhary, P. (2016). Illegal Economy vs. Socialist Society. International Journal of Research in Commerce \& Management, 7(12), 28-32.

CSNU. S/RES/1267. Octubre 15, 1999. https://www.un.org/securitycouncil/s/res/1267-\%281999\%29

CSNU. Security Council, Resolution 1373 on Threats to international peace and security caused by terrorist acts. 2001. https://www.un.org/ruleoflaw/blog/document/security-council-resolution-13732001-on-threats-to-international-peace-and-security-caused-by-terrorist-acts/

Cox, D. (2014). Handbook of Anti Money Laundering. Wiley.

Dawn Ivory, R. (2013). The Right to a Fair Trial and International Cooperation in Criminal Matters: Article 6 ECHR and the Recovery of Assets in Grand Corruption Cases. Utrecht Law Review, 9(4), 147-164. https://doi.org/10.18352/ulr.247

De Courcelles, D. (2009). La conformidad: historia filosófica y paradoja profética. Estudios: Filosofía, Historia, Letras, 9o(7), 30-46. https://doi.org/10.5347/01856383.0090.000174438

Enweremadu, D. U. (2013). Nigeria's Quest to Recover Looted Assets: The Abacha Affair. Africa Spectrum, 48(2), 51-70. https://doi.org/10.1177/000203971304800203

Fijnaut, C. (2013). The European Parliament and organised crime: the impending failure of the Alfano Committee. European Journal of Crime, Criminal Law and Criminal Justice, 21, 1-7. https://doi.org/10.1163/15718174-20170001

Füss, R. y Hecker, A. (2008). Profiling White-Collar Crime: Evidence from GermanSpeaking Countries. Corporate Ownership \& Control, 5(4), 149-161. https://doi.org/10.22495/cocv5i4p14

Via Inveniendi Et Iudicandi e-ISSN: 1909-0528 | DOI: https://doi.org/10.15332/19090528 Vol. 16 N.o 2 | julio-diciembre del 2021 
Gong, X. (2013). International Joint Action against Corruption: Agreement between China and Canada regarding the Sharing of Forfeited Assets. Journal of East Asia \& International Law, 6(2), 594-596.

Goyal, K. A. y Singh, V. (2019). Demonetization in India-The Journey So Far. Productivity, 6o(2), 195-203.

Ionescu, L. (2010). The Differential Effect of Corruption on Corporate Political Connections. Economics, Management, and Financial Markets, 5(4), 202-207.

Ionescu, L. (2012a). Corruption and Political Change. Economics, Management, and Financial Markets, 7(2), 155-160.

Ionescu, L. (2012b). Money Laundering Directives and Corruption in the European Union. Contemporary Readings in Law and Social Justice, 4(2), 562-566. https://www.ceeol.com/search/article-detail?id=25194

Jackson, P. (2005). Briefing: The Commission for Africa, Gleneagles, Brussels and Beyond. African Affairs, 104(417), 657-664. https://doi.org/10.1093/afraf/adio83

Jourdan, C., Hesler, T. y Calapa, A. (2008). Treasury functions driving compliance effectiveness: A focus on anti-money laundering and the Foreign Corrupt Practices Act. Journal of Corporate Treasury Management, 1(3), 238-248.

Kang, S. (2018). Rethinking the Global Anti-Money Laundering Regulations to Deter Corruption. International and Comparative Law Quarterly, 67, 695-720. https://doi.org/10.1017/So020589318000106

Kishor, N. y Lescuyer, G. (2012). Controlling illegal logging in domestic and international markets by harnessing multi-level governance opportunities. International Journal of the Commons, 6(2), 255-270. https://doi.org/10.18352/ijc.327

Kostyuchenko, N., Starinskyi, M., Tiutiunyk, I. y Kobushko, I. (2018). Methodical Approach to the Assessment of Risks Connected with the Legalization of the Proceeds of Crime. Montenegrin Journal of Economics, 14(4), 23-43.

https://doi.org/10.14254/1800-5845/2018.14-4.2

Kumar Mishtra, J. (2017). Why Repeat the Failed Experiment? The Logic \& the Economic Cost of the Note Ban. Optimization: Journal of Research in Management, 9(2), $53-61$.

Via Inveniendi Et Iudicandi

e-ISSN: 1909-0528 | DOI: https://doi.org/10.15332/19090528

Vol. 16 N.o 2 | julio-diciembre del 2021 
Levi, M. (2014). Money Laundering. En L. Paoli (ed.), The Oxford Handbook of Organized Crime (pp.419-443). Oxford University Press.

Lohaus, M. (2019). Towards a global consensus against corruption: International Agreements as Products of Diffusion and Signals of Commitment. Routledge. https://doi.org/10.4324/9780429492235

Marat, E. (2015). Global money laundering and its domestic political consequences in Kyrgyztan. Central Asian Survey, 34(1), 46-56. DOI: https://doi.org/10.1080/02634937.2015.1010854

Masciandaro, D., Takats, E. y Unger, B. (2007). Black finance: the economics of money laundering. Edward Elgar.

Mosquera, L. y González, A. (2020). Las brechas socioeconómicas y su relación con la corrupción: elementos clave a la hora de formular políticas públicas. Revista IUSTA, 52, 103-122. DOI: https://doi.org/10.15332/25005286.5485

Obuah, E. (2010). Combatting Corruption in Nigeria: The Nigerian Economic and Financial Crimes (EFCC). African Studies Quarterly, 12(1), 17-44.

Oji, R. O., Eme, O. I. y Onyishi, A. O. (2014). Implications of Politically Exposed Persons participation in 2015 Elections in Nigeria. Mediterranean Journal of Social Sciences, 5(27), 207-221. https://doi.org/10.5901/mjss.2014.v5n27p207

ONU. (1988). United Nations Convention against Illicit Traffic in Narcotic Drugs and Psychotropic Substances. 21 de diciembre de 1988. https://www.unodc.org/unodc/en/treaties/illicit-trafficking.html?ref=menuside

Pacini, C. (2012). The Foreign Corrupt Practices Act: Taking a Bite out of Bribery in International Business Transactions. Fordham Journal of Corporate \& Financial Law, 17, 545-589.

Pahontu, A. (2010). Fighting Against Organized Crime and the Action's Influence on the National Security Enforcement. Buletin Stiinfic, 15(2), 170-176.

Palomares, J. y Calonje, N. (2015) Tratados de libre comercio Colombia-Asia: Cuestión preliminar y perfiles de negociación. Revista IUSTA, 43(1), 17-41. https://doi.org/10.15332/s1900-0448.2015.0043.06

Quirk, P. (1996). Macroeconomic implications of money laundering. IMF Working Paper 96/66. FMI.

Via Inveniendi Et Iudicandi

e-ISSN: 1909-0528 | DOI: https://doi.org/10.15332/19090528

Vol. 16 N.o 2 | julio-diciembre del 2021 
Quirk, P. (1997). Money Laundering: Muddying the Macro-economy. Finance and Development, 1, 7-9.

Regis Prado, L. (2013). El nuevo tratamiento penal del blanqueo de capitales en el derecho brasileño (Ley 12.683/2012). Revista de Derecho Penal y Criminología, 3(10), 379-412.

Robertson, S. (2006). Misunderstanding Corruption. Anthropology Today, 22(2), 8-11.

Salgado, S., Oceja, L., Beramendi, M. y González, C. (2018). Towards a model of normative diagnosis in the organizational context: The evaluative model of normative appeals. Intangible Capital, 14(3), 484-497.

https://doi.org/10.3926/ic.1232

Savona, E. y Riccardi, M. (2015). From Illegal Markets to Legitimate Businesses: The Portfolio of Organised Crime in Europe - Final Report of Project OCP Organized Crime Portfolio. Transcrime - Università degli Studi di Trento.

Sharman, J. C. y Chaikin, D. (2009). Corruption and Anti-Money-Laundering Systems: Putting a Luxury Good to Work. Governance: An International Journal of Policy, Administration, and Institutions, 22(1), 27-45. https://doi.org/10.1111/j.14680491.2008.01420.x

Stessens, G. (2000). Money laundering: a new international law enforcement model. Cambridge University Press. https://doi.org/10.1017/CBO9780511494567

Sumaiya, F. (2018). Demonetization and its Impact on Indian Economy. International Journal of Research in Commerce \& Management, 9(1), 20-23.

Tanzi, V. (1996). Money laundering and the international financial system. FMI.

Unger, B., Rawlings, G., Siegel, M., Ferwerda, J., de Kruijf, W., Busuioic, E. M. y Wokke, K. (2006). The amounts and the effects of money laundering. Reporte al Ministerio Neerlandés de Finanzas.

Unger, B. y Busuioic, E. M. (2007). The scale and impacts of money laundering. Edward Elgar.

Ungerman, J. O. (2013). IRS CI Background and Current Initiatives. Journal of Tax Practice and Procedure, 27-58.

Via Inveniendi Et Iudicandi

e-ISSN: 1909-0528 | DOI: https://doi.org/10.15332/19090528

Vol. 16 N.० 2 | julio-diciembre del 2021 
UNODC (2000). United Nations Convention Against Transnational Organized Crime and the Protocols Thereto. 15 de noviembre de 2000.

https://www.unodc.org/unodc/en/organized-crime/intro/UNTOC.html

Van Dijk, J. (2007). Mafia markers: assessing organized crime and its impact upon societies. Trends in Organized Crime, 10(39), 39-56.

https://doi.org/10.1007/s12117-007-9013-X

Van Duyne, P.C. (2003). Money laundering policy: fears and facts. En Van Duyne, P.C., Von Lampe, K. y Newell, J.L. (eds.). Criminal finances and organising crime in Europe. Nijmegen: Wolf Legal Publishers.

Van Duyne, P.C. y Nelemans, M.D.H. (2011). Thinking in and out of Plato's cave. En Allum, F. y Gilmour, S. (eds.). Routledge Handbook of Transnational Organized Crime. Routledge. https://doi.org/10.4324/9780203698341

Van Duyne, P. C., Harvey, J. H. y Gelemerova, L. Y. (2018) (eds.). The Critical Handbook of Money Laundering. Policy, Analysis \& Myths. Palgrave Macmillan.

Villaromán, N. G. (2012). Recovering the Proceeds of Corruption in the Philippines: A Comparison between the Unexplained Wealth Act (R.A. $n{ }^{\circ}{ }^{1379}$ ) and the AntiMoney Laundering Act (R.A. No. 9160, as Amended). Ateneo Law Journal, $57(746), 746-766$.

Walker, J. (1995). Estimates of the extent of money laundering in and through Australia. Paper prepared for the Australian Transaction Report and Analysis Centre. John Walker Consulting Centre.

Walker, J. y Unger, B. (2009). Measuring Global Money Laundering: 'The Walker Gravity Model'. Review of Law and Economics, 5(2), 821-853.

https://doi.org/10.2202/1555-5879.1418

Zali, M. y Maulidi, A. (2018). Fighting Against Money Laundering. BRICS Law Journal, 5(3), 40-63. https://doi.org/10.21684/2412-2343-2018-5-3-40-63

Zdanowicz, J. S. (2009). Trade-Based Money Laundering and Terrorist Financing. Review of Law and Economics, 5(2), 855-878. https://doi.org/10.2202/15555879.1419

Via Inveniendi Et Iudicandi

e-ISSN: 1909-0528 | DOI: https://doi.org/10.15332/19090528

Vol. 16 N.० 2 | julio-diciembre del 2021 